\title{
A Chemically Programmed Proximal Ligand Enhances the Catalytic Properties of a Heme Enzyme
}

\author{
Anthony P. Green, ${ }^{*}{ }^{\dagger, \#}$ Takahiro Hayashi, ${ }^{\ddagger}, \#$ Peer R. E. Mittl, ${ }^{\S}$ and Donald Hilvert ${ }^{*}+$ \\ ${ }^{\dagger}$ School of Chemistry \& Manchester Institute of Biotechnology, The University of Manchester, 131 Princess Street, Manchester M1 \\ 7DN, U.K \\ ${ }^{\ddagger}$ Laboratory of Organic Chemistry, ETH Zurich, 8093 Zürich, Switzerland \\ ${ }^{\S}$ Department of Biochemistry, University of Zürich, 8057 Zürich, Switzerland
}

\section{Supporting Information}

ABSTRACT: Enzymes rely on complex interactions between precisely positioned active site residues as a mechanism to compensate for the limited functionality contained within the genetic code. Heme enzymes provide a striking example of this complexity, whereby the electronic properties of reactive ferryl intermediates are finely tuned through hydrogen bonding interactions between proximal ligands and neighboring amino acids. Here, we show that introduction of a chemically programmed proximal $N_{\delta}$-methyl histidine (NMH) ligand into an engineered ascorbate peroxidase (APX2) overcomes the reliance on the conserved Asp-His hydrogen bonding interaction, leading to a catalytically modified enzyme (APX2 $\mathrm{NMH}$ ), which is able to achieve a significantly higher number of

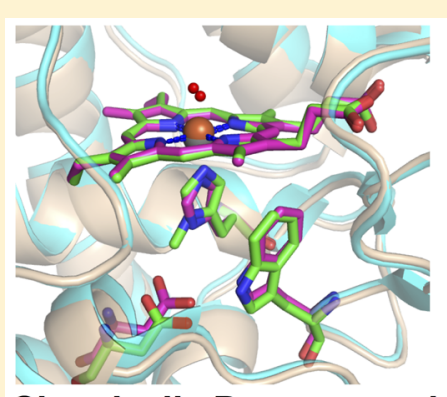

Chemically Programmed Heme Ligand

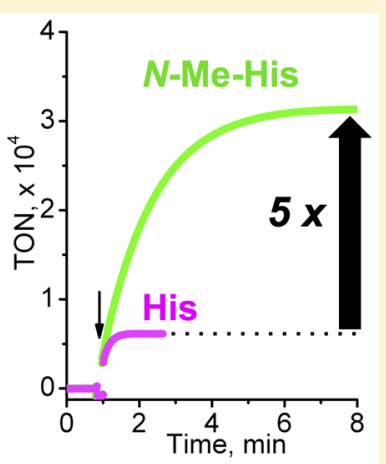
turnovers compared with APX2 without compromising catalytic efficiency. Structural, spectroscopic and kinetic characterization of APX2 NMH and several active site variants provides valuable insights into the role of the Asp-His-Fe triad of heme peroxidases. More significantly, simplification of catalytic mechanisms through the incorporation of chemically optimized ligands may facilitate efforts to create and evolve new active site heme environments within proteins.

\section{INTRODUCTION}

Enzymes are able to mediate a diverse array of energetically demanding transformations using a limited set of functional amino acids presented by the genetic code. The enormous rate enhancements provided by these biological catalysts are achieved through the evolution of sophisticated catalytic machineries that typically rely on interactions between precisely positioned networks of amino acids. Considerable effort has been devoted to understanding the catalytic roles played by the individual functional amino acids across the different enzyme classes. ${ }^{1}$ Perhaps not surprisingly, such analysis reveals that histidine is the most frequently encountered and versatile catalytic residue, due to its ability to serve as both a general acid and base, ${ }^{2}$ a coordinating ligand in metalloenzymes, ${ }^{3}$ and as nucleophilic residue in mechanisms involving covalent intermediates. ${ }^{4}$ Due to the presence of two nitrogen atoms in its imidazole ring, histidine is able to participate in multiple interactions with substrates, cofactors and neighboring functional residues simultaneously. For example, the conserved AspHis-Ser triad of serine proteases ${ }^{2}$ and the Asp-His-Fe triad of heme peroxidases ${ }^{5,6}$ are among the most studied catalytic motifs in biochemistry (Figure 1a). While the primary function of the central histidine residue in such systems is often well established, defining the precise role played by secondary interactions with neighboring residues during catalysis presents a formidable challenge.

Mutation of an amino acid that is intimately involved in an enzyme's catalytic mechanism typically results in dramatic reduction in activity, providing a simple method of identifying essential residues. However, the limited range of functionality present within the canonical amino acids severely restricts the degree of mechanistic information that can be uncovered using this approach. In the present study, we demonstrate how expansion of the genetic code to include structural analogues of histidine provides an effective strategy to probe key secondary interactions required for efficient catalysis in a heme enzyme. Such fundamental understanding is essential to advance our ability to rationally re-engineer existing enzymes or design biocatalysts with novel function.

Heme peroxidases utilize hydrogen peroxide as an electron acceptor to catalyze a range of oxidative transformations that have been exploited for biotechnological applications. ${ }^{7}$ These enzymes utilize a histidine residue as the axial ligand of hemeiron, with a hydrogen bond formed between the noncoordinating $N_{\delta}$ atom and an aspartate residue. ${ }^{8,9}$ This AspHis-Fe triad is conserved across the heme peroxidase family

Received: July 7, 2016

Published: August 8, 2016 
a

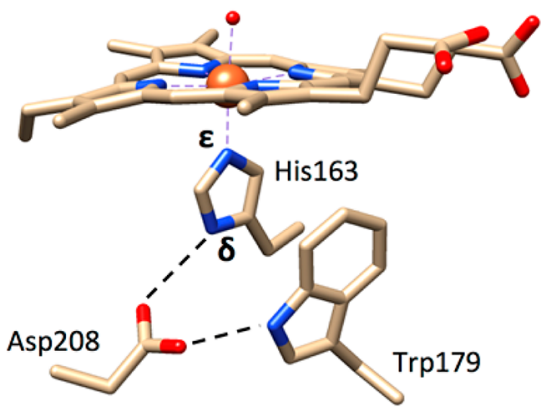

b

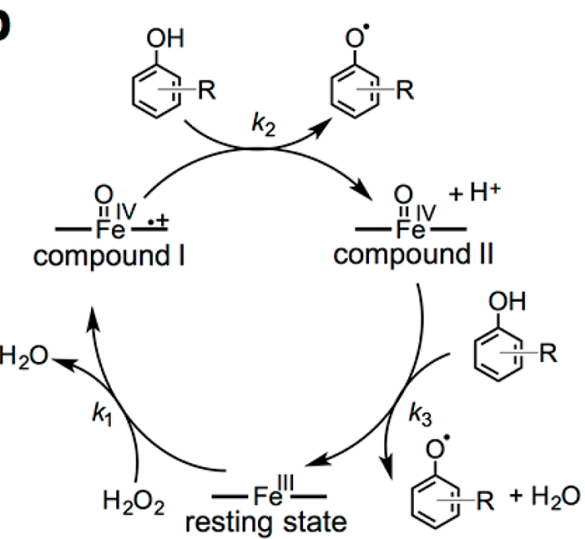

Figure 1. Structure and catalytic mechanism of ascorbate peroxidase. (a) The active site of ascorbate peroxidase (PDB ID: 1OAG). ${ }^{31}$ (b) The catalytic cycle of heme peroxidases with a phenolic substrate as the electron donor.

(Figure 1a) and is reminiscent of the Asp-His-Ser catalytic triad of serine proteases ${ }^{10}$ and the Asp-His-Zn motif of a number of metalloenzymes. ${ }^{11}$ In contrast, the aspartate residue is not present in other heme proteins containing axial histidine residues, including myoglobin and hemoglobin which possess low levels of promiscuous peroxidase activity. ${ }^{12}$ Despite extensive theoretical and experimental study, the precise role of the Asp-His hydrogen-bonding interaction remains poorly understood. This interaction is widely claimed to impart partial imidazolate character onto the axial ligand, which in turn has been proposed to assist in $\mathrm{O}-\mathrm{O}$ bond heterolysis, stabilize key ferryl-oxo intermediates, and fine-tune the redox potential/ electronic properties of the $\mathrm{Fe}$ center (Figure 1b)., ${ }^{5,6,9,13}$ Calculations performed on imidazole and imidazolate ligated ferryl porphyrin species suggest that the peroxidase Asp-His hydrogen bond modifies the electronic structure of compound I intermediates by promoting delocalization of the porphyrin radical onto the proximal histidine ligand, ${ }^{14-17}$ although this has yet to be experimentally validated.

With the exception of a relatively conservative Asp $\rightarrow$ Glu substitution, mutation of the conserved aspartate residue in peroxidases results in dramatic reduction in catalytic activity. ${ }^{13}$ However, in addition to its hydrogen bonding interaction with the axial histidine ligand, the essential aspartate residue may play a role in electrostatic stabilization of positive charge and participate in additional interactions with other proximal residues. For example, in the well-studied cytochrome $c$ peroxidase (CCP) and ascorbate peroxidase (APX), this aspartate residue forms an additional hydrogen bond with a neighboring tryptophan which in turn participates in a $\pi$ stacking interaction with the proximal histidine. ${ }^{18}$ Remarkably, despite the similar active site architectures of CCP and APX, the electronic properties of the compound I intermediate of these two enzymes differ significantly, with a proximal Trp191 centered radical cation formed in CCP compared with a porphyrin $\pi$ cation radical in APX. ${ }^{19-21}$ In short, the complex network of interactions formed by the proximal aspartate residue make it challenging to define the importance of the HisAsp hydrogen bond by simple mutation of this conserved amino acid.

Expansion of the genetic code to include a range of histidine analogues has recently been described, ${ }^{22}$ presenting an opportunity to study the significance of the Asp-His hydrogen-bonding interaction from a different angle, namely by direct replacement of the axial histidine ligand with structurally related analogues. A number of features make $N_{\delta}$-methyl histidine $(\mathrm{NMH})$ a particularly attractive residue to probe AspHis interactions. First, the absence of the $N_{\delta}$ hydrogen means that $\mathrm{NMH}$ is incapable of forming a hydrogen bond to the aspartate residue. Due to the comparable $\mathrm{p} K_{\mathrm{a}}$ values associated with imidazole and $N$-methylimidazole (7.18 and 7.38, respectively), ${ }^{23}$ relatively minor differences in electron donating properties of proximal histidine and $\mathrm{NMH}$ ligands are expected in the absence of additional interactions. Finally, the alkyl modification effectively forces the imidazole ring to adopt a single tautomeric form and prevents the formation of "imidazolate like" proximal ligands (via loss or polarization of the $N_{\delta}$ hydrogen), thus overcoming ambiguity surrounding imidazole charge state which complicates interpretation of catalytic mechanisms involving histidine. ${ }^{14}$ Here, we demonstrate that substitution of the proximal histidine with $\mathrm{NMH}$ in an engineered ascorbate peroxidase (APX2) results in a modified enzyme with enhanced catalytic properties, despite disrupting the "essential" Asp-His hydrogen bonding interaction which is conserved across the peroxidase family.

\section{RESULTS}

An engineered variant of ascorbate peroxidase ${ }^{24}$ was selected as a model enzyme for proximal ligand replacement (Figure 2a). The selective incorporation of NMH into proteins in response to amber codons was recently described using an evolved pyrrolysyl-tRNA synthetase/pyrrolysl-tRNA (PylRS(NMH)/ tRNA $^{\text {Pyl }}$ ) pair. ${ }^{22}$ Two copies of the relevant PylRS variant were subcloned into the pEVOL plasmid which has been optimized for the production of proteins containing noncanonical residues. ${ }^{25}$ Coexpression of this pEVOL construct with APX2 (AMBER) on a standard pET29b vector led to the production of the desired APX2 variant, with a protein yield of $>50 \mathrm{mg}$ per liter of culture after purification (see Experimental Procedures). Stoichiometric replacement of the proximal histidine residue (His163) with NMH was confirmed by MS analysis of the intact protein as well as peptide fragments generated by tryptic digestion (Figure S1). The UV-vis spectrum of His163NMH APX2 (APX2 NMH) in the resting state is indistinguishable from that of the wild-type enzyme, suggesting that this proximal ligand modification causes minimal changes to the electronic and structural properties of the heme iron (Figure $2 \mathrm{~b}$ ). Both variants are characterized by a Soret maximum at $404 \mathrm{~nm}$ with associated Q bands at 500/632 $\mathrm{nm}$ and $540 / 574 \mathrm{~nm}$, consistent with the presence of a mixture of six-coordinated (6c) high-spin (HS) and low-spin (LS) heme iron. ${ }^{26}$ The heme occupancy of purified APX2 and APX2 $\mathrm{NMH}$ variants is also very similar, as judged by the ratio of intensities of the bands at 404 and $280 \mathrm{~nm}$. Addition of cyanide 


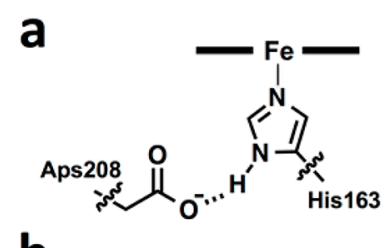

b
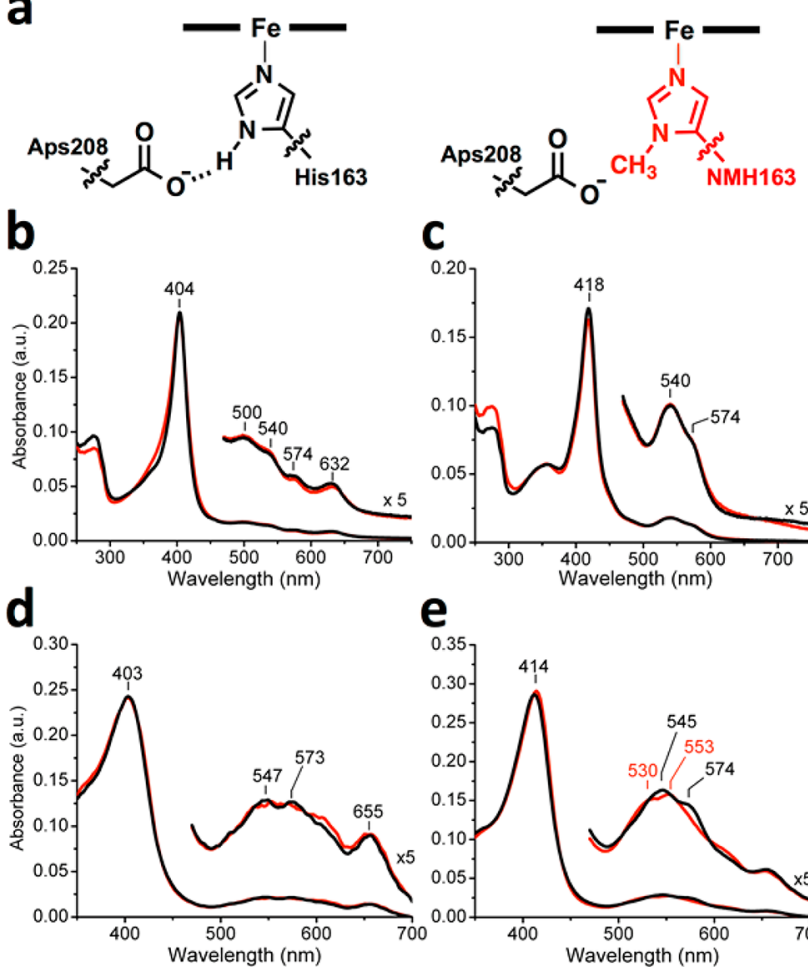

C
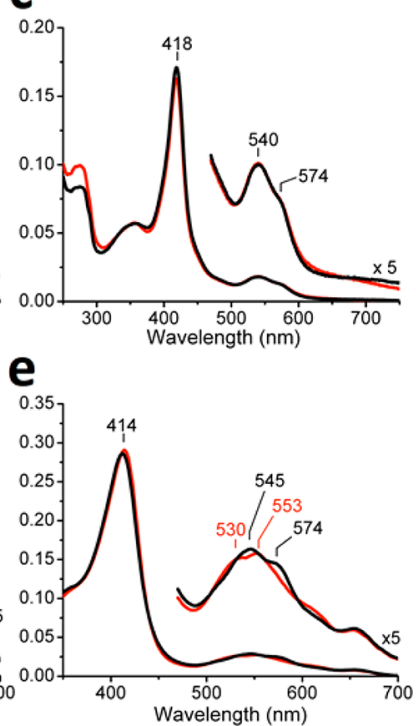

Figure 2. Characterization of APX2 and APX2 NMH. (a) Replacement of the proximal histidine ligand (His163) by NMH disrupts the hydrogen-bonding network in the proximal pocket. (b-e) Overlay of the UV-vis spectra of APX2 (black) and APX2 NMH (red) ferric resting state (b), CN-bound state (c), compound I (d) and compound II (e).

Scheme 1. Oxidation of Guaiacol Mediated by APX2

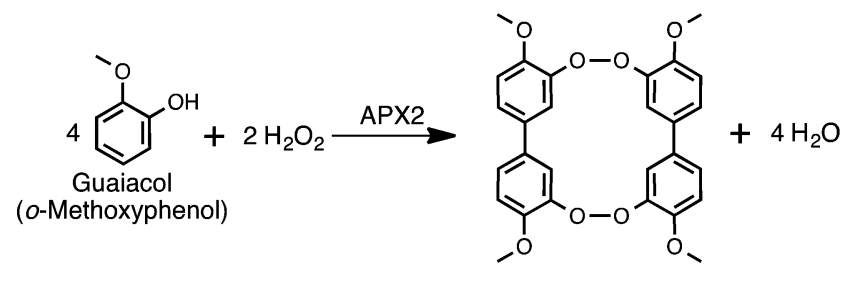

to the resting enzymes results in similar spectral changes in APX2 and APX2 NMH due to the formation of 6c-LS species (Figure 2c). Compounds I and II (Figure 1b) have been implicated as common intermediates in many proteins. Although these species are highly unstable in enzymes like the cytochrome P450s, ${ }^{27}$ they are readily detected in APX under relatively mild conditions. ${ }^{28}$ The UV-vis spectra of compound I (Figure 2d) and compound II (Figure 2e) intermediates of APX2 and APX2 NMH were therefore recorded following mixing of the resting enzymes with hydrogen peroxide in the absence of phenolic substrate. ${ }^{28}$ Only minor differences are observed between the spectral features of these key APX2 and APX2 NMH ferryl intermediates, suggesting that the proximal His $\rightarrow \mathrm{NMH}$ substitution does not lead to significant changes in the electronic structures of compound I and compound II.

The catalytic parameters of the APX2 and APX2 NMH variants for the oxidation of the peroxidase substrate $o$ methoxyphenol (guaiacol) in the presence of hydrogen peroxide $(2.5 \mathrm{mM})$ were determined using a stopped-flow apparatus. Peroxidase-mediated guaiacol oxidation leads to the formation of a stable tetrameric species whose formation can be readily monitored by absorbance at $470 \mathrm{~nm}$ (Scheme 1). This assay has been exploited previously for the characterization of APX2, with an optimal $\mathrm{H}_{2} \mathrm{O}_{2}$ concentration between 1.0 and 10 $\mathrm{mM}$ reported. ${ }^{24}$ Remarkably, despite disruption of the conserved Asp-His hydrogen bonding interaction, introduction of a proximal NMH ligand results in a slight increase in catalytic efficiency, with $k_{\text {cat }} / K_{\mathrm{m}}$ values of $8.6 \times 10^{4} \mathrm{M}^{-1} \mathrm{~s}^{-1}\left(K_{\mathrm{m}}=36 \pm\right.$ $5 \mathrm{mM}$ and $\left.k_{\text {cat }}=3060 \pm 360 \mathrm{~s}^{-1}\right)$ and $1.1 \times 10^{5} \mathrm{M}^{-1} \mathrm{~s}^{-1}\left(K_{\mathrm{m}}=\right.$ $14 \pm 1 \mathrm{mM}$ and $\left.k_{\mathrm{cat}}=1500 \pm 90 \mathrm{~s}^{-1}\right)$ determined for APX2 and APX2 NMH, respectively (Figure 3a). Furthermore, in the

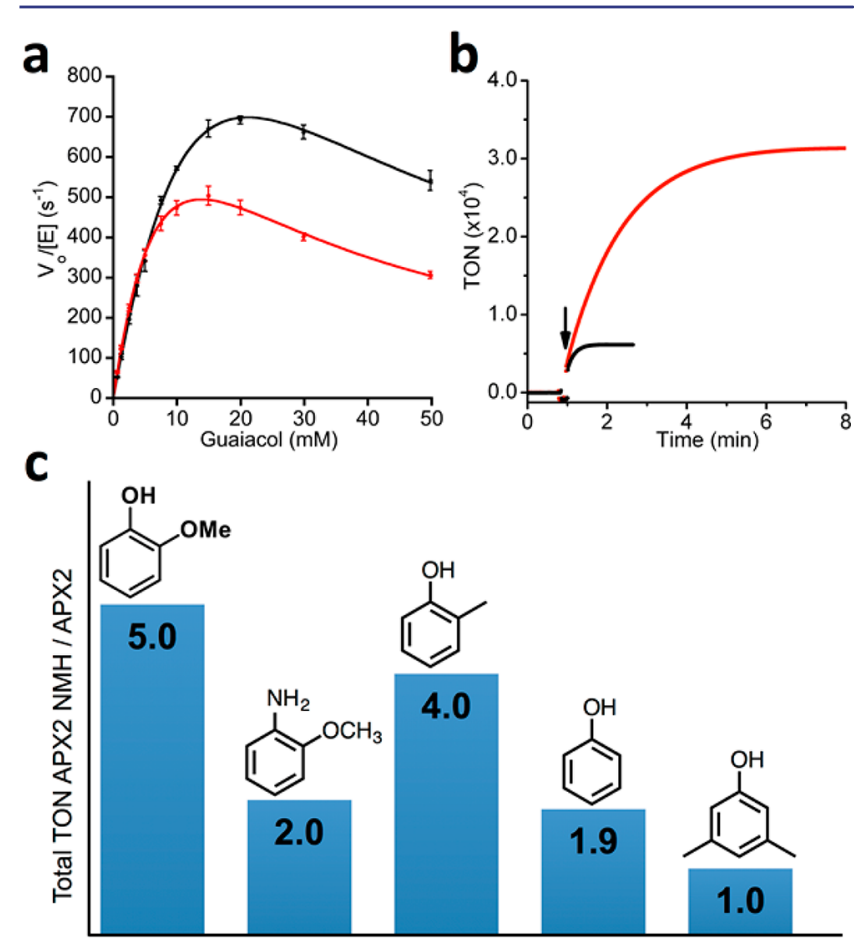

Figure 3. Catalytic properties of APX2 and APX2 NMH. (a) Michaelis-Menten plots and (b) time course of guaiacol oxidation by APX2 (black) and APX2 NMH (red). Formation of the stable tetrameric product was monitored at 470 or $540 \mathrm{~nm}$. TON is given by moles of product formed per mole of enzyme. The black arrow indicates the time of enzyme addition. APX2 and APX2 NMH were able to achieve 6200 and 31300 turnovers, respectively, prior to irreversible inactivation. (c) Comparison of total TON of oxidation of various phenolic substrates by APX2 and APX2 NMH.

absence of substrate under presteady-state conditions, only minor differences in the rate constants for compound $\mathrm{I}\left(k_{1}\right)$ and compound II $\left(k_{2}\right)$ formation were determined for $\operatorname{APX} 2\left(k_{1}=\right.$ $\left.1.4 \times 10^{9} \mathrm{M}^{-1} \mathrm{~s}^{-1}, k_{2}=1.2 \times 10^{6} \mathrm{M}^{-1} \mathrm{~s}^{-1}\right)$ and $\mathrm{APX} 2 \mathrm{NMH}\left(k_{1}\right.$ $=6.0 \times 10^{8} \mathrm{M}^{-1} \mathrm{~s}^{-1}, k_{2}=6.1 \times 10^{5} \mathrm{M}^{-1} \mathrm{~s}^{-1}$ ) (Figure S2).

Determination of the total turnovers achieved by APX2 and APX2 NMH reveals a more dramatic difference in their catalytic behavior. Heme peroxidases are known to suffer irreversible inactivation during catalysis (phenol oxidation), ${ }^{29}$ a significant drawback when potential industrial applications are considered. ${ }^{30}$ In our hands, APX2 was able to promote guaiacol oxidation with a total turnover number (TON) of 6200 prior to inactivation, a value comparable to that reported previously, albeit under slightly modified conditions (Figure $3 b){ }^{24}$ In contrast, a TON of 31300 was determined for APX2 NMH, which represents a 5 -fold increase as a result of the His $\rightarrow$ $\mathrm{NMH}$ proximal ligand mutation (Figure $3 \mathrm{~b}$ ). The UV-vis 

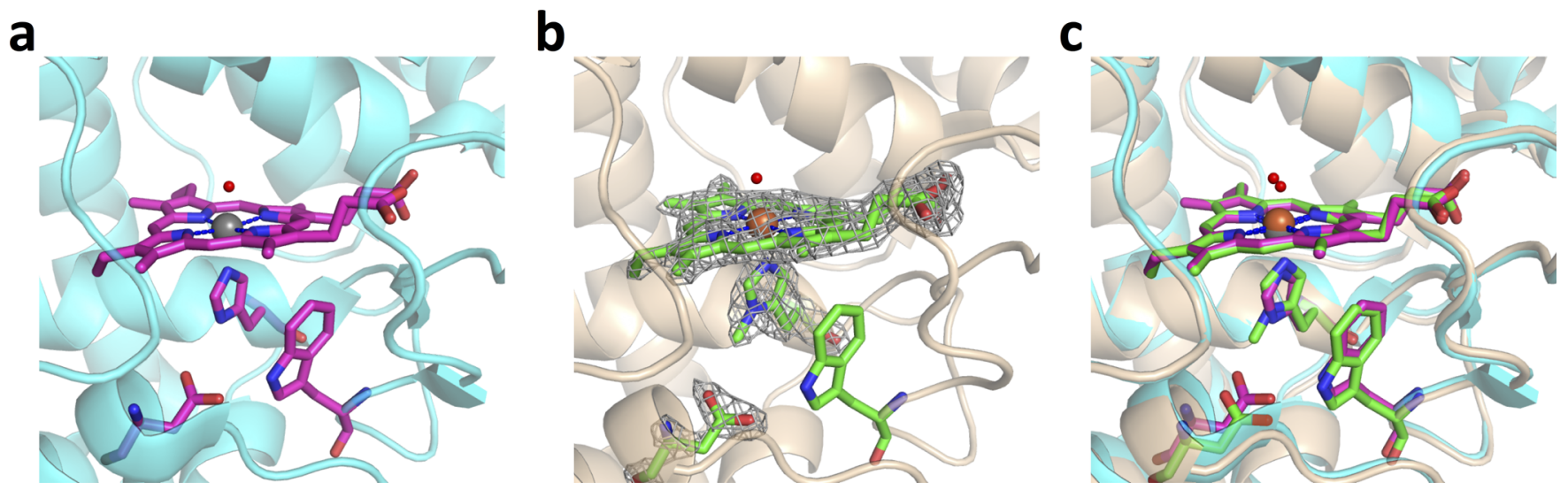

Figure 4. Crystal structures of APX and APX2 NMH. (a) The active site of APX (PDB ID: 1OAG). ${ }^{31}$ The heme cofactor and the proximal His163, Trp179 and Asp208 are shown in atom colored sticks (carbon, nitrogen and oxygen colored in magenta, blue and red, respectively) with the remainder of the protein showed in ribbon (colored cyan). An ordered water molecule is present in the distal pocket binding the heme iron (shown as a red sphere). (b) The active site of APX2 NMH. The heme cofactor and the proximal NMH163, Trp179 and Asp208 are shown in atom colored sticks (carbon, nitrogen and oxygen colored in green, blue and red, respectively) with the remainder of the protein showed in ribbon (colored in brown). The omit maps for the heme cofactor, NMH163 and Asp208 shown as gray mesh, contoured at 2.5 $\sigma$. An ordered water molecule is present in the distal pocket binding the heme iron (shown as a red sphere). (c) Overlay of the active sites of APX and APX2 NMH to minimize the rmsd between the heme cofactors. The heme cofactors, active site residues and ordered water molecules of APX and APX2 NMH are shown in atom colored sticks, color coded as in panels a and $\mathrm{b}$, respectively.

spectra of the product formed by the APX2 and APX2 NMH variants are identical, confirming that the same product is formed during both transformations. Significantly higher TONs were also observed for oxidation of $o$-anicidine, $o$-cresol, and phenol by NMH APX2 compared with APX2 (Figure 3c).

The crystal structure of APX2 NMH was solved and refined to a resolution of $1.9 \AA$ (Table S2). This structure superimposes well with a previously reported APX structure, ${ }^{31}$ with little movement of the peptide backbone ( $\mathrm{rmsds}$ of 0.21 and $0.39 \AA$ for the superposition of chains $\mathrm{A}$ and $\mathrm{B}$, respectively, on $1 \mathrm{OAG}$ ) and no significant changes in location of the iron cofactor or the heme plane (Figure 4). In order to accommodate the additional methyl substituent on the proximal histidine, the helix containing Asp208 was displaced by $\sim 1.0 \AA$. The $\mathrm{N}-\mathrm{Fe}$ distance to the coordinating axial ligand is $1.95 \AA$, compared with the value of $2.02 \AA$ reported previously for APX in its resting state. ${ }^{31}$ An iron-bound water in the distal pocket is also well ordered. These structural details show that the high catalytic efficiency and TONs achieved by

Table 1. Catalytic Parameters for Asp208 Variants of APX2 and APX2 NMH $^{a}$

\begin{tabular}{|c|c|c|c|c|c|}
\hline variant & $\begin{array}{l}k_{\text {cat }} \\
\left(\mathrm{s}^{-1}\right)\end{array}$ & $\begin{array}{c}K_{\mathrm{M}} \\
(\mathrm{mM})\end{array}$ & $\begin{array}{c}K_{\mathrm{I}} \\
(\mathrm{mM})^{b}\end{array}$ & $\underset{\left(\mathrm{mM}^{-1} \mathrm{~s}^{-1}\right)}{k_{\mathrm{ct}} / K_{\mathrm{M}}}$ & $\begin{array}{l}\text { total } \\
\text { TON }\end{array}$ \\
\hline APX2 & 3060 & 36.0 & 12.5 & 86 & 6200 \\
\hline APX2 D208N & 8.8 & 1.5 & 25 & 6.0 & 600 \\
\hline APX2 D208M & 88.7 & 19.0 & 103 & 4.7 & 1000 \\
\hline APX2 NMH & 1500 & 14.0 & 14.1 & 110 & 31300 \\
\hline $\begin{array}{l}\text { APX2 NMH } \\
\text { D208A }\end{array}$ & 194 & 11.0 & 74.8 & 18 & 7000 \\
\hline $\begin{array}{l}\text { APX2 NMH } \\
\text { D208N }\end{array}$ & 351 & 18.5 & 44.9 & 19 & 9600 \\
\hline APX2 NMH & 780 & 25.9 & 34.1 & 30 & 18900 \\
\hline
\end{tabular}

${ }^{a}$ All measurements were carried out at $25{ }^{\circ} \mathrm{C}$ in PBS containing 2.5 $\mathrm{mM} \mathrm{H} \mathrm{H}_{2} \mathrm{O}_{2}$ and $5 \mathrm{nM}$ enzyme. ${ }^{b}$ Inhibitory constant $K_{\mathrm{I}}$ was derived from the Michaelis-Menten equation for uncompetitive substrate inhibition.
APX2 NMH do not arise as a result of major structural reorganization within the active site. The $\mathrm{NMH}$ residue adopts a similar conformation to histidine residues in previously reported structures. As a result, the proximal $\mathrm{NMH}$ maintains $\pi$-stacking interactions with Trp179 and the newly introduced methyl substituent points directly toward Asp208, which also maintains a similar conformation to that observed in a previously reported resting state APX structure. ${ }^{31}$

To determine whether Asp208 remains an important functional residue in the $\mathrm{NMH}$-containing peroxidase (Figure 2a), D208A and D208N mutations were introduced into both APX2 and APX2 NMH. The D208A variant of APX2, which was difficult to produce, failed to bind the heme cofactor to any appreciable extent as judged by UV-vis spectroscopy, and attempts to reconstitute it with free heme cofactor in vitro gave only very low amounts of the holoenzyme. Introduction of the D208N mutation into APX2 led to a dramatic decrease in catalytic efficiency and TON (Figure 5 and Table 1). In contrast, the D208A and D208N APX2 NMH variants retained significant levels of catalytic activity (D208A $k_{\text {cat }} / K_{\mathrm{m}}=1.8 \times$ $10^{4} \mathrm{M}^{-1} \mathrm{~s}^{-1}$, D208N $\left.k_{\text {cat }} / K_{\mathrm{m}}=1.9 \times 10^{4} \mathrm{M}^{-1} \mathrm{~s}^{-1}\right)$, albeit reduced compared with the starting $\mathrm{APX} 2 \mathrm{NMH}$ variant $\left(k_{\mathrm{cat}} /\right.$ $K_{\mathrm{m}}=1.1 \times 10^{5} \mathrm{M}^{-1} \mathrm{~s}^{-1}$ ) (Figure S3). Significantly, despite mutation of the Asp208 residue, these variants were able to perform a comparable or slightly higher number of total turnovers than APX2 prior to deactivation (TONs: APX2 NMH/D208A, 7000; APX2 NMH/D208N, 9600).

To identify the optimal residue at position 208 in APX2 $\mathrm{NMH}$, a library was generated using NNK codons and evaluated using a whole cell microtiter-plate assay based on guaiacol oxidation. In addition to three silent mutations, an Asp $\rightarrow$ Met mutation was identified which performed only a slightly reduced number of total turnovers compared with APX2 NMH in the whole cell assay (Figure S4). Purification and characterization of this APX2 NMH D208M variant revealed only a modest reduction in catalytic efficiency and TONs compared with APX2 NMH $\left(k_{\text {cat }} / K_{\mathrm{m}}=3.0 \times 10^{4} \mathrm{M}^{-1} \mathrm{~s}^{-1}\right.$, TON; 18900 ) (Figure 5 and $S 3$ ). These parameters were improved compared with the D208N NMH and D208A NMH 


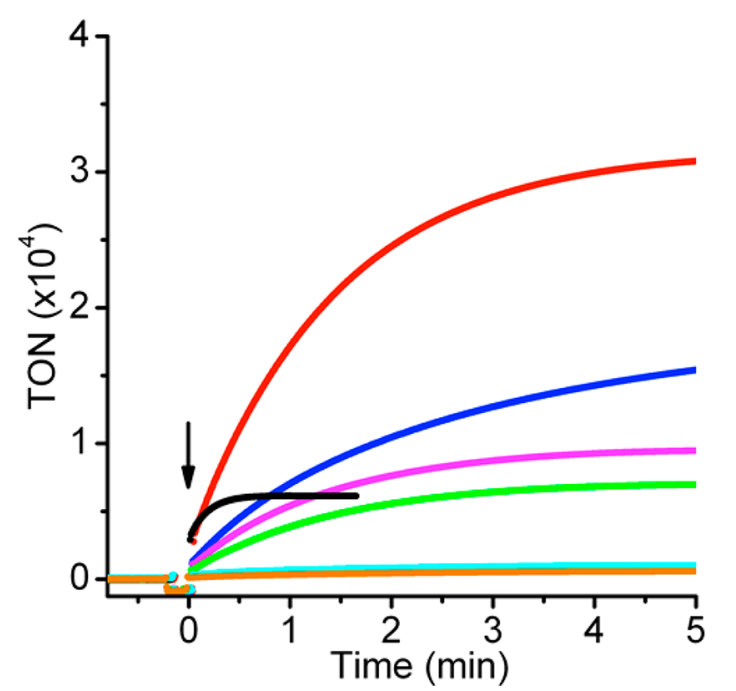

Figure 5. Characterization of Asp208 variants of APX2 and APX2 NMH. Time course of guaiacol oxidation by APX2 (black), APX2 D208N (orange), APX2 D208M (cyan), APX2 NMH (red), APX2 NMH D208A (green), APX2 NMH D208N (magenta) and APX2 NMH D208M (blue). Formation of the stable tetrameric product was monitored at $540 \mathrm{~nm}$. TON is given by moles of product formed per mole of enzyme. The black arrow indicates the time of enzyme addition.

variants, and significantly higher than the corresponding APX2 D208M variant $\left(k_{\mathrm{cat}} / K_{\mathrm{m}}=4.7 \times 10^{3} \mathrm{M}^{-1} \mathrm{~s}^{-1}\right.$, TON; 1000) (Figure 5 and S3). Interestingly, a methionine residue has been identified at the corresponding position of dehaloperoxidase hemoglobin A (DHP A). ${ }^{32}$ In this enzyme, one of the sulfur lone pairs is in contact with the proximal histidine ligand which has been proposed to increase the electron donating character of the histidine through a charge transfer interaction. It is conceivable that the methionine residue performs a similar role in the APX2 NMH D208M variant. Combined, these data suggest that replacement of the axial histidine ligand with $\mathrm{NMH}$ confers a greater degree of mutational flexibility at position 208 . Despite the fact that it is no longer able to participate in a hydrogen-bonding interaction with the proximal ligand, aspartate still remains the optimal residue at position 208 . We speculate that this residue may stabilize partial positive charge that develops on the axial histidine ligand through electrostatic interactions or, alternatively, play a role in optimizing the $\pi-\pi$ interaction between $\operatorname{Trp} 179$ and the proximal ligand.

In contrast to APX2 and APX2 NMH which display indistinguishable spectral features, comparison of the UV-vis spectra of the D208X variants reveal clear differences (Figure S5). Mutation of Asp208 in APX2 NMH causes displacement of the Soret maximum from 404 to $408 \mathrm{~nm}$. The four Q bands (at 502/633 nm and 540/576 nm) associated with 6c-HS and $6 \mathrm{c}$-LS heme iron, respectively, are evident in all D208X variants investigated (D208M, D208N and D208A), albeit with a reduced 6c-HS:6c-LS ratio compared with the starting Asp208 variant. In contrast, introduction of the corresponding point mutations into APX2 (D208N and D208M) causes displacement of the Soret to $411 \mathrm{~nm}$, loss of two Q bands associated with the $6 \mathrm{c}-\mathrm{HS}$ species, and the emergence of a new band at $669 \mathrm{~nm}$ which is also present to a lesser extent in the UV-vis spectra of selected APX2 NMH D208X variants. These data suggest that the Asp208 residue may play a key role in stabilizing the catalytically productive electronic structure of the heme iron in APX2 which is less critical in APX2 NMH.

\section{DISCUSSION}

Considerable effort has been devoted to unraveling the precise mechanistic role played by conserved Asp-His hydrogen bonding interactions ${ }^{33-35}$ which are prevalent catalytic motifs found in diverse classes of metallo- and non-metalloenzymes. Suggested roles include controlling the conformation of the histidine side chain, stabilizing the appropriate histidine tautomer, stabilizing positively charged histidine intermediates formed along the reaction coordinate during catalysis, perturbing the $\mathrm{p} K_{\mathrm{a}}$ of the histidine residue, or a combination of the above. Specifically in the case of heme peroxidases, the Asp-His interaction is reported to impart partial imidazolate character onto the axial ligand which may assist in $\mathrm{O}-\mathrm{O}$ bond heterolysis or perturb the electronic properties of the iron center. $^{5,6,9,13}$

In this study, disruption of the Asp-His hydrogen bond by replacement of the proximal histidine ligand with the noncanonical structural analogue NMH has allowed us to reexamine the mechanistic role of this conserved interaction in heme peroxidases. Replacement of the $N_{\delta}$ hydrogen of histidine with an alkyl residue has a number of structural/functional consequences which directly relate to previously suggested roles for Asp-His interactions. This modification (1) effectively fixes the tautomeric form of the imidazole ring; (2) ensures the neutral charge state of the proximal ligand throughout the catalytic cycle (i.e., formation of the anionic ligand via deprotonation of the $N_{\delta}$ hydrogen is not possible); and (3) prevents buildup of imidazolate character through $\mathrm{N}-\mathrm{H}$ bond polarization, thus overcoming ambiguity surrounding imidazole charge state. The comparable resting state UV-vis spectra and rates of compound I formation in APX2 and APX2 NMH suggest that any contribution made by the proximal Asp-His hydrogen bond (in $\mathrm{APX} 2$ ) to $\mathrm{H}_{2} \mathrm{O}_{2}$ heterolysis or to the electronic properties of the heme cofactor (resting state) is compensated by the minor increase in basicity associated with the NMH ligand. It can be assumed that the tautomeric form of the APX2 histidine ligand is controlled by coordination to the Fe center (assuming that the histidine ligand is in the neutral form). Furthermore, comparison of the X-ray structure of APX2 NMH and previously reported APX structures reveals only minor structural changes which occur to accommodate the methyl substituent. The orientation of the histidine and $\mathrm{NMH}$ ligands is almost identical in these structures, suggesting that the Asp-His interaction is not required to control the conformation of the histidine side chain. Thus, comparison of the kinetic parameters, spectroscopic properties and X-ray structures has allowed us to exclude a number of plausible mechanistic roles for the Asp-His hydrogen-bonding interaction.

So how can we explain the increased "robustness" of APX2 $\mathrm{NMH}$ toward irreversible inactivation compared with APX2, and how does this relate to the role of the proximal pocket AspHis hydrogen-bonding interaction? Several hypotheses for peroxidase deactivation mechanisms have been proposed. ${ }^{36,37}$ Our study shows that small structural/electronic changes in the proximal pocket can profoundly influence these processes. In agreement with the aforementioned theoretical studies, ${ }^{14-17}$ we suggest that the Asp-His interaction may play a role in stabilizing the reactive ferryl intermediates, potentially by promoting partial delocalization of the porphyrin radical of 
compound I onto the proximal histidine ligand. In that case, the introduction of a proximal $\mathrm{NMH}$ ligand (in APX2 NMH) would provide a more effective and robust means of stabilizing compound I that is not reliant on a noncovalent hydrogenbonding interaction which could potentially be susceptible to uncoupling throughout repeated catalytic cycles. The electrondonating methyl substituent on the noncoordinating $N_{\delta}$ atom is expected to increase radical stability through hyperconjugation, thus favoring radical delocalization onto the proximal $\mathrm{NMH}$ residue. This mechanistic interpretation is consistent with the observation that APX2 NMH is tolerant to mutations at Asp208, while such modifications in APX2 lead to dramatic reductions in catalytic activity and total turnover number.

What is evident from the present study is that the direct targeting of "essential" catalytic residues for substitution by chemically programmed amino acids provides a fruitful avenue to probe enzyme mechanism ${ }^{38-42}$ and to produce biocatalysts with enhanced properties. This is in contrast to traditional enzyme engineering approaches, where canonical substitutions of catalytic amino acids often lead to a dramatic loss of enzyme activity. In this study, disruption of the catalytic Asp-His-Fe triad of a heme peroxidase by introduction of a proximal $\mathrm{NMH}$ ligand led to biocatalysts with comparable catalytic efficiencies to the "wild type" enzyme but with increased robustness toward irreversible inactivation during catalysis (i.e., increase in total turnover numbers). Natural evolutionary processes have clearly not optimized the proximal pocket to support this chemically modified residue, and it would be interesting to observe whether laboratory evolution can further enhance the catalytic parameters of the modified enzyme. Perhaps equally significant is the increased mutational tolerance at Asp208 as a result of introducing a chemically modified residue. This finding demonstrates how nature relies upon the evolution of complex interactions between catalytic residues as a mechanism to compensate for the limited functionality contained within the genetically encoded amino acids. Simplification of catalytic mechanisms through the genetic incorporation of chemically programmed amino acids ${ }^{40-42}$ will certainly enhance our understanding of the minimum requirements for catalyst design and should facilitate efforts to create and evolve new active site chemistries/environments within proteins. ${ }^{43}$

\section{EXPERIMENTAL PROCEDURES}

Materials. All chemical and biological reagents were purchased from commercial suppliers and were used without further purification. LB medium and 2xYT were purchased from MP Biomedicals, LLC (California, USA.) and Sigma-Aldrich (Missouri, USA). Hemin chloride and $30 \%$ hydrogen peroxide were purchased from Merck Millipore (Darmstadt, Germany), $\delta$-aminolevulinic acid from Acros Organics (Geel, Belgium), guaiacol and lysozyme from Sigma-Aldrich, 3-methyl-L-histidine (NMH) from Bachem (Bubendorf, Switzerland), DNase I from Roche Diagnosis GmbH (Mannheim, Germany). Phusion High-Fidelity DNA polymerase, T4 DNA ligase and all restriction enzymes used in this study were purchased from New England BioLabs (Massachusetts, USA). Oligonucleotides were synthesized by Microsynth AG (Balgach, Switzerland). A plasmid encoding pcDNA3 APX2-NES was a kind gift from Prof. Alice Ting (Addgene plasmid \# 49386). ${ }^{24}$ pBK-PylRS and pEVOL tRNApyl/ pylRSWT were kind gifts from Prof. Jason Chin and Prof. Edward Lemke, respectively.

Physical and Biophysical Measurements. UV-vis absorption spectra were acquired on a Lamda 35 spectrophotometer (PerkinElmer, Massachusetts, USA). Fast protein liquid chromatography (FPLC) was carried out on an NGC Quest 10 plus chromatography system (Biorad laboratories, California, USA) equipped with a multiwavelength detector and BioFrac fraction collector. Sodium dodecyl sulfate-polyacrylamide gel electrophoresis (SDS-PAGE) was performed on a PhastSystem electrophoresis apparatus using precast PhastGel homogeneous 12.5 (GE healthcare Life Sciences, Chicago, USA). Matrix-assisted laser ionization time-of-flight mass spectrometry (MALDI-TOF-MS) was performed on a microflex (Bruker Daltonics, Bremen, Germany) using $\alpha$-cyano-4-hydroxycinnamic acid (CHCA) or sinapinic acid (SA) as a matrix. High resolution mass spectra were recorded on an electrospray ionization quadrupole time-of-flight (ESIQ-TOF) system MaXis (Bruker Daltonics, Bremen, Germany) coupled with an Agilent 1200 system (Agilent technology, California, USA). Steady-state and presteady-state stopped-flow experiments were performed on an Applied Photophysics SX18 stopped-flow spectrophotometer (Applied Photophysics Ltd., Leatherhead, UK) equipped with a xenon arc lamp and a $1 \mathrm{~cm}$ path length.

Construction of pET29_APX2, APX2 H163TAG and D208X Variants. The gene encoding APX2 was amplified from pcDNA3 APX2-NES (Addgene plasmid \# 49386) ${ }^{24}$ using the primer pair APX2_NdeI_for and APX2_XhoI_rev (Table S1). The resulting DNA fragment was digested by NdeI and XhoI and ligated into a pET29b(+) vector digested by the same restriction enzymes, yielding pET29b_APX2. The codon for His163 in pET29b_APX2 was replaced by an amber codon (TAG) by overlap extension PCR. Briefly, two fragments corresponding to the $\mathrm{N}$ and $\mathrm{C}$ termini were synthesized with the primer pairs APX2_NdeI_for and H163TAG_rev, H163TAG_for and APX2_XhoI_rev (Table S1). The two $\mathrm{P} \overline{\mathrm{C} R}$ products were purified and then combined in an equimolar ratio for final PCR. The full length fragment was assembled using the primer pair APX2_NdeI_for and APX2_XhoI_rev. The mutated fragment was digested $\bar{b}$ NdeI and $\bar{X} h o I$ and ligated into a pET29b(+) as described above, yielding pET29b_APX2 H163TAG. The Asp208 mutations were introduced into the pET29b_APX2 and pET29b_APX2 H163TAG by overlap extension PCR. Two fragments corresponding to the $\mathrm{N}$ and $\mathrm{C}$ termini were synthesized with the primer pairs APX2_NdeI_for and D208_rev, D208A_for, D208N_for or D208M_for and APX 2 _XhoI_rev. The $\mathrm{N}$ and $\bar{C}$ termini $\overline{\mathrm{PCR}}$ products were purified and then combined in an equimolar ratio for final PCR. The full length fragments were assembled using the primer pair APX2_NdeI_for and APX2_XhoI_rev. The mutated fragment was digested by $\overline{\mathrm{N}}$ deI and $\mathrm{XhoI}$ and ligated into a pET29b(+) as described above. The DNA sequence was confirmed by DNA sequencing service provided by Microsynth AG.

Construction of pEVOL_PyIRS(NMH). Five mutations (L207I, Y271F, L274G, C313F, and Y349F) were introduced into the pBKPylRS plasmid generate pBKPylRS(NMH) as described previously. ${ }^{22}$ The Y349F point mutation was first introduced by overlap extension PCR. Fragments corresponding to the $\mathrm{N}$ and $\mathrm{C}$ termini were synthesized using the primer pairs PylRS for and Y349F_rev, Y349F_for and PylRS_rev (Table S1). The products of the two previous PCRs were purified and then combined in equimolar amounts for the final PCR. The full-length fragment was amplified using the primer pair PylRS_for and PylRS_rev (Table S1). The mutated fragment was digested by NdeI and $\bar{P} s t \mathrm{I}$ and ligated into a pBK vector digested by the same restriction enzymes, yielding pBK PylRS Y349. Three fragments corresponding to the $\mathrm{N}$ terminus, midd̄e, and $\mathrm{C}$ terminus were synthesized using PylRS_for and L270IY271FL274G_rev, L270IY271FL274G_for and C313F_rev, C313F_for and PylRS_rev (Table S1). The three PCR products were purified and then combined in equimolar amounts for the final PCR. The full length fragment was assembled using a primer pair PylRS_for and PylRS_rev. The mutated fragment was digested by NdeI and Pst I and ligated into a $\mathrm{pBK}$ vector digested by the same restriction enzymes, yielding pBK_PylRS(NMH). To construct pEVOL_PylRS(NMH), the same mutated fragment digested by NdeI and PstI was initially ligated into a $\mathrm{pEVOL}$ vector digested by the same restriction enzymes. Next, the PylRS(NMH) gene was amplified using the primer pair pBK_PylRS(NMH)_BglII and pBK_PylRS(NMH)_SalI, digested with BglII and Sall, and ligated into the pEVOL vector digested by the same restriction enzymes to give pEVOL_PylRS(NMH) containing two copies of the PylRS(NMH) 
gene. The DNA sequence was confirmed as described above using pBAD_for, pBAD_rev, and two custom primers (pEVOLconst_for and $\mathrm{pEVOL}$ const rev).

Protein Production and Purification. For expression of APX2, pET29b_APX2 was transformed into BL21(DE3) E. coli and the cells were plated on a LB agar plate containing $50 \mu \mathrm{g} / \mathrm{mL}$ kanamycin. A single colony of freshly transformed cells was cultured overnight in 3 $\mathrm{mL}$ of $\mathrm{LB}$ medium containing $50 \mu \mathrm{g} / \mathrm{mL}$ kanamycin. One $\mathrm{mL}$ of the culture was used to inoculate $100 \mathrm{~mL}$ of $2 \mathrm{xYT}$ medium supplemented with $1 \mathrm{mM} \delta$-aminolevulinic acid and $50 \mu \mathrm{g} / \mathrm{mL}$ kanamycin. The culture was incubated for $\sim 2 \mathrm{~h}$ at $37{ }^{\circ} \mathrm{C}$ with shaking speed at 230 $\mathrm{rpm}$. When the $\mathrm{OD}_{600}$ of the culture reached $\sim 0.5$, IPTG was added to a final concentration of $0.1 \mathrm{mM}$ to induce overexpression of the APX2 gene. APX2 D208X variants were produced using this same procedure. For expression of APX2 NMH, pET29b_APX2 H163TAG and pEVOL_PylRS(NMH) were transformed into BL21(DE3) E. coli and the cells were plated on a LB agar plate containing $50 \mu \mathrm{g} / \mathrm{mL}$ kanamycin and $34 \mu \mathrm{g} / \mathrm{mL}$ chloramphenicol. A single colony of freshly transformed cells was cultured overnight in $3 \mathrm{~mL}$ of $\mathrm{LB}$ medium containing $50 \mu \mathrm{g} / \mathrm{mL}$ kanamycin and $34 \mu \mathrm{g} / \mathrm{mL}$ chloramphenicol. One $\mathrm{ml}$ of the culture was used to inoculate $100 \mathrm{~mL}$ of $2 \mathrm{xYT}$ medium supplemented with $1 \mathrm{mM} \delta$-aminolevulinic acid, $12 \mathrm{mM} \mathrm{NMH,} 50$ $\mu \mathrm{g} / \mathrm{mL}$ kanamycin and $34 \mu \mathrm{g} / \mathrm{mL}$ chloramphenicol. The culture was incubated for $\sim 2.5 \mathrm{~h}$ at $37^{\circ} \mathrm{C}$ with shaking at $230 \mathrm{rpm}$. When the $\mathrm{OD}_{600}$ of the culture reached $\sim 0.5$, IPTG and arabinose were added to a final concentration of $0.1 \mathrm{mM}$ and $0.05 \%$, respectively. APX2 NMH D208X variants were produced using this same procedure. The induced cultures were incubated for $\sim 24 \mathrm{~h}$ at $25^{\circ} \mathrm{C}$, and the cells were subsequently harvested by centrifugation at $5500 \mathrm{~g}$ for $15 \mathrm{~min}$. The pelleted bacterial cells were suspended in $50 \mathrm{mM}$ Tris- $\mathrm{HCl}, 300 \mathrm{mM}$ $\mathrm{NaCl}$ at pH8.0 (buffer A) with $10 \mathrm{mM}$ imidazole containing $0.5 \%$ lysozyme and $0.01 \%$ DNase and sonicated to disrupt the cells. The lysate was centrifuged at $11500 \mathrm{~g}$ for $15 \mathrm{~min}$ and the supernatant was subjected to affinity chromatography using Ni-NTA Agarose (Qiagen, Helden, Germany). His-tagged APX2 and APX2 NMH were eluted by buffer A containing $300 \mathrm{mM}$ imidazole. To maximize heme occupancy, partially purified APX2 and APX2 NMH were reconstituted with hemin chloride. Briefly, Ni-NTA purified protein solutions were rapidly mixed with one equivalent of hemin chloride dissolved in 10 $\mathrm{mM} \mathrm{NaOH}$, and incubated at room temperature for $1 \mathrm{~h}$. The APX2 and APX2 NMH solutions were then subjected to size-exclusion (SEC) chromatography on Superdex 200 10/300 GL (GE healthcare Life Sciences) with a flow rate of $0.5 \mathrm{~mL} / \mathrm{min}$ using an NGC Quest 10 plus FPLC system equilibrated with PBS $\left(10 \mathrm{mM} \mathrm{Na}_{2} \mathrm{HPO}_{4}, 1.8 \mathrm{mM}\right.$ $\mathrm{KH}_{2} \mathrm{PO}_{4}, 2.7 \mathrm{mM} \mathrm{KCl}$, and $\left.137 \mathrm{mM} \mathrm{NaCl}, \mathrm{pH} 7.4\right)$. The fractions were monitored at 260,280 , and $408 \mathrm{~nm}$, collected using a BioFrac fraction collector, and analyzed by SDS-PAGE. If necessary, the protein solution was concentrated by Amicon Ultra-15 10-kDa cutoff centrifugal filter device (Merck Millipore, Massachusetts, USA). The concentrations of heme-containing proteins were determined using an extinction coefficient of $128000 \mathrm{M}^{-1} \mathrm{~cm}^{-1}$ at $405 \mathrm{~nm}^{24}$

MS Analysis of APX2 and APX2 NMH. MS data for APX2 (Figure S1a) and APX2 NMH (Figure S1b) were acquired on an ESIQ-TOF system coupled with an Agilent 1200 system. The protein solutions were desalted by Zeba spin desalting column (ThermoFisher Scientific, Massachusetts, USA) equilibrated with pure water containing $0.1 \%$ formic acid, and the final protein concentrations were adjusted to $1 \mathrm{mg} / \mathrm{mL}$. The MS instrument was operated in wide pass quadrupole mode with the TOF data being collected between $\mathrm{m} /$ $z$ 100-5000 with low-collision energy of $8 \mathrm{eV}$. The optimized source conditions were drying gas $8.0 \mathrm{l} / \mathrm{h}$ (nitrogen $99.99 \%$ purity) at a temperature of $200^{\circ} \mathrm{C}$, nebulizer pressure $1.6 \mathrm{bar}$, capillary and end plate voltages 500 and $4500 \mathrm{~V}$, respectively, TOF flight tube voltage $9880 \mathrm{~V}$, reflection voltage $2004 \mathrm{~V}$, pusher voltage $1640 \mathrm{~V}$ and MCP detector voltage $2927 \mathrm{~V}$. The ESI-TOF mass spectrometer was calibrated routinely for flow injection analysis (FIA) in the positive electrospray ionization mode using Agilent-ESI-TOF tuning mix on the enhanced quadratic algorithmic mode. Further data processing was calculated using the Data Analysis 4.1 software (Bruker Daltonics) with deconvolution software MaxEnt (Spectrum Square Associates,
USA). The peptide fragment $149-170$ of APX2 and APX2 NMH was analyzed to confirm the stoichiometric replacement of the His 163 residue with NMH (Figure S1c). To the purified proteins $(100 \mu \mathrm{M})$ were added urea $(2 \mathrm{M})$ and trypsin (proteomics grade, Sigma-Aldrich) at $10 \mathrm{wt} \%$, followed by incubation at $37^{\circ} \mathrm{C}$ overnight. The generated peptide fragments were analyzed by MALDI-MS (Bruker Daltonics).

Spectroscopic Characterization of APX2 and APX2 NMH Variants. UV-vis absorption spectra of APX2 and APX2 NMH variants in the resting state were recorded on a Lamda 35 spectrophotometer in PBS buffer $\left(10 \mathrm{mM} \mathrm{Na} \mathrm{HPO}_{4}, 1.8 \mathrm{mM}\right.$ $\mathrm{KH}_{2} \mathrm{PO}_{4}, 2.7 \mathrm{mM} \mathrm{KCl}$, and $\left.137 \mathrm{mM} \mathrm{NaCl}, \mathrm{pH} 7.4\right)$ at a final concentration of 2-5 $\mu \mathrm{M}$. The CN-bound APX2 and APX2 NMH were obtained following addition of sodium cyanide at the final concentration of $1 \mathrm{mM}$ in PBS (Figure 2c). Compound I and II of APX2 and APX2 NMH (Figure 2d, e) were generated as previously described. ${ }^{28}$ Briefly, APX2 and APX2 NMH were diluted to $10 \mu \mathrm{M}$ on the basis of the heme absorption $\left(\varepsilon_{405}=128000 \mathrm{M}^{-1} \mathrm{~cm}^{-1}\right)$ in PBS. PBS buffer containing 20 or $500 \mu \mathrm{M} \mathrm{H}_{2} \mathrm{O}_{2}$ was prepared and used immediately. Solutions were loaded into the stopped-flow UV-vis spectrometer equilibrated to $25{ }^{\circ} \mathrm{C}$. Compound I formation was complete in $\sim 50 \mathrm{~ms}$ for a 1:2 ratio of enzyme and hydrogen peroxide. Compound II formation was complete in $\sim 500 \mathrm{~ms}$ for a 1:50 ratio of enzyme and hydrogen peroxide.

Determination of Total Turnover Numbers. Reactions were carried out at $25^{\circ} \mathrm{C}$ in PBS in $1 \mathrm{~mL}$ quartz cuvettes containing $10 \mathrm{mM}$ guaiacol and $2.5 \mathrm{mM} \mathrm{H}_{2} \mathrm{O}_{2}$. Reactions were initiated by adding freshly purified APX2 and APX2 NMH at the final concentration of $5 \mathrm{nM}$. Product formation was monitored by the change in absorbance at 540 $\mathrm{nm}\left(\varepsilon_{540}=8209 \mathrm{M}^{-1} \mathrm{~cm}^{-1}\right)$.

Stopped-Flow Kinetics. APX2, APX2 NMH and D208X variants were diluted to $10 \mathrm{nM}$ on the basis of the heme absorption $\left(\varepsilon_{405}=\right.$ $128000 \mathrm{M}^{-1} \mathrm{~cm}^{-1}$ ) in PBS. Substrate solutions containing 1.3, 2.5, 5.0, $7.5,10,15,20,30,40,60$, and $100 \mathrm{mM}$ guaiacol and $5 \mathrm{mM} \mathrm{H}_{2} \mathrm{O}_{2}$ were prepared in PBS and used immediately. Solutions were loaded into the stopped-flow UV-vis spectrometer equilibrated to $25^{\circ} \mathrm{C}$. The product formation was monitored by the change in absorbance at $470 \mathrm{~nm}$. Typically, 3-5 shots were averaged and the averaged traces were fitted by linear regression using Kaleidagraph. The observed initial rates were fitted to the Michaelis-Menten equation for uncompetitive substrate inhibition (Figure 3a for APX2 and APX2 NMH; Figure S3 for APX2 NMH D208M, D208N, D208A, and APX2 D208M, D208N). Pseudofirst-order rate constants for compound I formation in APX2 and APX2 NMH were obtained as follows. Briefly, one syringe of the stopped-flow apparatus containing $2 \mu \mathrm{M}$ enzyme in PBS was mixed with the other containing at least a 10 -fold excess of $\mathrm{H}_{2} \mathrm{O}_{2}(20,30,40$, and $50 \mu \mathrm{M})$ in the same buffer. The second order rate constants were extracted from the slope of the plots. ${ }^{28}$ Compound I formation was monitored by a decrease in absorbance at $398 \mathrm{~nm}$. Similarly, compound II formation was monitored by an increase in absorbance at $424 \mathrm{~nm}$ following mixing of $4 \mu \mathrm{M}$ enzyme in PBS with an equal volume of $300 \mu \mathrm{M} \mathrm{H}_{2} \mathrm{O}_{2}$ in the same buffer.

Substrate Scope of APX2 and APX2 NMH. Reactions were carried out at $25^{\circ} \mathrm{C}$ in PBS in $1 \mathrm{~mL}$ quartz cuvettes containing $10 \mathrm{mM}$ substrates and $2.5 \mathrm{mM} \mathrm{H} \mathrm{H}_{2}$. Reactions were initiated by adding freshly purified APX2 and APX2 NMH at the final concentration of 5 $\mathrm{nM}$. Product formation was monitored by the change in absorbance at 480,410 , and $420 \mathrm{~nm}$ for $o$-anisidine, $o$-cresol, and 3,5dimethylphenol, respectively. The relative number of turnovers performed by APX2 NMH and APX2 are shown in Figure 3c. For the experiments with phenol, a stopped-flow UV-vis spectrometer was used due to the relatively rapid decay of the products formed. Briefly, APX2 and APX2 were diluted to $10 \mathrm{nM}$ on the basis of the heme absorption in PBS. Substrate solutions containing $20 \mathrm{mM}$ phenol and $5 \mathrm{mM} \mathrm{H}_{2} \mathrm{O}_{2}$ were prepared in PBS and used immediately. Solutions were loaded into the stopped-flow UV-vis spectrometer equilibrated to $25^{\circ} \mathrm{C}$. Product formation was monitored by the change in absorbance at $420 \mathrm{~nm}$. The relative number of turnovers performed by APX2 NMH and APX2 are shown in Figure 3c.

Crystallization and Structure Determination. APX2 NMH in PBS buffer was concentrated to approximately $20 \mathrm{mg} / \mathrm{mL}$ in an 
Amicon Ultra-15 $10-\mathrm{kDa}$ cutoff centrifugal filter device. The protein solution was passed through a PD-10 column (GE Healthcare Life Sciences) pre-equilibrated with pure water, and the final concentration was adjusted to $15 \mathrm{mg} / \mathrm{mL}$. The protein was crystallized by vapor diffusion in sitting drops at $20{ }^{\circ} \mathrm{C}$. An initial crystallization hit was identified in a well containing $100 \mathrm{mM}$ Bis-Tris at pH 5.5 and $2.0 \mathrm{M}$ ammonium sulfate using a commercial sparse matrix screen (JCSG+ Suite, Qiagen). Crystallization conditions were improved by varying the buffer $\mathrm{pH}$ and ammonium sulfate concentration. The optimal crystallization condition was $100 \mathrm{mM}$ bis-tris at pH 7.0 containing 2.0 $\mathrm{mM}$ ammonium sulfate. Crystals were cryoprotected by plunging into mother liquor supplemented with $20 \% \mathrm{w} / \mathrm{v}$ glycerol prior to flash freezing in liquid nitrogen. Diffraction data were collected at $100 \mathrm{~K}$ using a wavelength of $1.00 \AA$ at the Swiss Light Source (SLS) X06SA PX beamline. Maximum resolution of diffraction was estimated according to the literature. ${ }^{44}$ The structure was solved by molecular replacement with MOLREP using the ascorbate peroxidase structure (PDB ID: 1VOH) The structure was further refined using the programs REFMAC5 $^{45}$ and COOT. ${ }^{46}$ Refinement statistics are summarized in Supplementary Table S2.

APX2 NMH D208X Library Construction and Screening. Saturation mutagenesis using NNK codons was employed to randomize residue 208 of APX2 NMH. This was achieved by overlap extension PCR using pET29b_APX2 H163TAG as the template. Two fragments corresponding to the $\mathrm{N}$ and $\mathrm{C}$ termini were synthesized with APX2-NdeI-for and D208_rev, D208NNK_for and APX2-XhoIrev. The products of the two previous PCRs were purified and then combined in equimolar amounts for final PCR. The full length library was amplified using a primer pair APX2-NdeI-for and APX2-XhoI-rev. The gene fragment containing degenerate codons was digested by NdeI and XhoI and ligated into a pET29b(+) as described above. The library was cotransformed with pEVOL_PylRS(NMH) into BL21(DE3) E. coli. Redundancy in the random library was eliminated by picking 88 freshly transformed colonies from each library, and the picked colonies were used to inoculate 96-well plates filled with 180 $\mu \mathrm{L}$ LB medium containing $50 \mu \mathrm{g} / \mathrm{mL}$ kanamycin and $34 \mu \mathrm{g} / \mathrm{mL}$ chloramphenicol. Freshly cotransformed colonies of pET29b_APX2 $\mathrm{NMH}$ and pEVOL_PylRS(NMH) were used to inoculate the remaining wells as "wild-type" controls. A $5 \mu \mathrm{L}$ aliquot of the culture was used to inoculate 96-well plates filled with $180 \mu \mathrm{L}$ of $2 \mathrm{xYT}$ medium containing $1 \mathrm{mM} \delta$-aminolevulinic acid $12 \mathrm{mM} \mathrm{NMH}, 50 \mu \mathrm{g} /$ $\mathrm{mL}$ kanamycin and $34 \mu \mathrm{g} / \mathrm{mL}$ chloramphenicol. The plate was incubated for $\sim 2.5 \mathrm{~h}$ at $37^{\circ} \mathrm{C}$ with shaking at $230 \mathrm{rpm}$. When the $\mathrm{OD}_{600}$ of the cultures reached $\sim 0.5$, IPTG and arabinose were added to a final concentration of $0.1 \mathrm{mM}$ and $0.05 \%$, respectively. The induced culture was incubated for $\sim 24 \mathrm{~h}$ at $25{ }^{\circ} \mathrm{C}$, and used for screening, which was carried out as follows: $5 \mu \mathrm{L}$ of resuspended cultures were transferred into 96-well plates filled with $100 \mu \mathrm{L}$ of PBS. To the cells was added $100 \mu \mathrm{L}$ of assay solution containing $3 \mathrm{mM}$ guaiacol and $2.5 \mathrm{mM} \mathrm{H}_{2} \mathrm{O}_{2}$ in PBS. Product formation was monitored by the change in absorbance at $470 \mathrm{~nm}$ (Figure S4).

\section{ASSOCIATED CONTENT}

\section{S Supporting Information}

The Supporting Information is available free of charge on the ACS Publications website at DOI: 10.1021/jacs.6b07029.

MS data, UV-vis spectral data, kinetic analysis, list of primers, and structure statistics (PDF)

\section{AUTHOR INFORMATION}

\section{Corresponding Authors}

*anthony.green@manchester.ac.uk

*hilvert@org.chem.ethz.ch

\section{Author Contributions}

${ }^{\#}$ A.P.G. and T.H. contributed equally.

\section{Notes}

The authors declare no competing financial interest.
The crystal structure of APX2 NMH was deposited in the RCSB Protein Data Bank (PDB) under accession number $5 \mathrm{~L} 86$.

\section{ACKNOWLEDGMENTS}

This research was generously supported by the Biotechnology and Biological Sciences Research Council (David Phillips Fellowship BB/M027023/1, APG), the Swiss National Science Foundation (31003A 156276, DH), ETH Zurich (DH) and a JSPS fellowship $(\bar{T} \bar{H})$. Plasmids pBK-PylRS and pEVOL tRNApyl/pylRSWT were kind gifts from Prof. Jason Chin and Prof. Edward Lemke. The authors are grateful to C. StutzDucommun and B. Blattmann for help with protein crystallization, and the beamline staff at the Swiss Light Source for support during data collection.

\section{REFERENCES}

(1) Holliday, G. L.; Mitchell, J. B.; Thornton, J. M. J. Mol. Biol. 2009, 390, 560-577.

(2) Polgár, L. Cell. Mol. Life Sci. 2005, 62, 2161-2172.

(3) Sundberg, R. J.; Martin, R. B. Chem. Rev. 1974, 74, 471-517.

(4) Rigden, D. J. Biochem. J. 2008, 409, 333-348.

(5) Poulos, T. L. JBIC, J. Biol. Inorg. Chem. 1996, 1, 356-359.

(6) Goodin, D. B. JBIC, J. Biol. Inorg. Chem. 1996, 1, 360-363.

(7) van Rantwijk, F.; Sheldon, R. A. Curr. Opin. Biotechnol. 2000, 11, 554-564.

(8) Poulos, T. L. Arch. Biochem. Biophys. 2010, 500, 3-12.

(9) Raven, E. L. Nat. Prod. Rep. 2003, 20, 367-381.

(10) Carter, P.; Wells, J. A. Nature 1988, 332, 564-568.

(11) Christianson, D. W.; Alexander, R. S. J. Am. Chem. Soc. 1989, $111,6412-6419$.

(12) Wan, L.; Twitchett, M. B.; Eltis, L. D.; Mauk, A. G.; Smith, M. Proc. Natl. Acad. Sci. U. S. A. 1998, 95, 12825-12831.

(13) Goodin, D. B.; McRee, D. E. Biochemistry 1993, 32, 3313-3324.

(14) Green, M. T. J. Am. Chem. Soc. 2000, 122, 9495-9499.

(15) deVisser, S. P.; Shaik, S.; Sharma, P. K.; Kumar, D.; Thiel, W. J. Am. Chem. Soc. 2003, 125, 15779-15788.

(16) Derat, E.; Cohen, S.; Shaik, S.; Altun, A.; Thiel, W. J. Am. Chem. Soc. 2005, 127, 13611-13621.

(17) Rydberg, P.; Sigfridsson, E.; Ryde, U. JBIC, J. Biol. Inorg. Chem. 2004, 9, 203-223.

(18) Finzel, B. C.; Poulos, T. L.; Kraut, J. J. Biol. Chem. 1984, 259, 13027-13036.

(19) Sivaraja, M.; Goodin, D. B.; Smith, M.; Hoffmann, B. M. Science 1989, 245, 738-740.

(20) Patterson, W. R.; Poulos, T. L.; Goodin, D. B. Biochemistry 1995, 34, 4342-4345.

(21) Bonagura, C. A.; Sundaramoorthy, M.; Pappa, H. S.; Patterson, W. R.; Poulos, T. L. Biochemistry 1996, 35, 6107-6115.

(22) Xiao, H.; Peters, F. B.; Yang, P.-Y.; Reed, S.; Chittuluru, J. R.; Schultz, P. G. ACS Chem. Biol. 2014, 9, 1092-1096.

(23) Gagliano, R. A., Jr; Knowlton, R. C.; Byers, L. J. Org. Chem. 1989, 54, 5247-5250.

(24) Lam, S. S.; Martell, J. D.; Kamer, K. J.; Deerinck, T. J.; Ellisman, M. H.; Mootha, V. K.; Ting, A. Y. Nat. Methods 2014, 12, 51-54.

(25) Young, T. S.; Ahmad, I.; Yin, J. A.; Schultz, P. G. J. Mol. Biol. 2010, 395, 361-374.

(26) Dalton, D. A.; Daiz del Castillo, L.; Kahn, M. L.; Joyner, S. L.; Chatfield, J. M. Arch. Biochem. Biophys. 1996, 328, 1-8.

(27) Rittle, J.; Green, M. T. Science 2010, 330, 933-937.

(28) Marquez, L. A.; Quitoriano, M.; Zilinskas, B. A.; Dunford, H. B. FEBS Lett. 1996, 389, 153-156.

(29) Huang, Q.; Huang, Q.; Pinto, R. A.; Griebenow, K.; SchweitzerStenner, R.; Weber, W. J., Jr. J. Am. Chem. Soc. 2005, 127, 1431-1437.

(30) van de Velde, F.; van Rantwijk, F.; Sheldon, R. A. Trends Biotechnol. 2001, 19, 73-80. 
(31) Sharp, K. H.; Mewies, M.; Moody, P. C.; Raven, E. L. Nat. Struct. Biol. 2003, 10, 303-307.

(32) LaCount, M. W.; Zhang, E.; Chen, Y. P.; Han, K.; Whitton, M. M.; Lincoln, D. E.; Woodin, S. A.; Lebioda, L. J. Biol. Chem. 2000, 275, 18712-18716.

(33) Frey, P.; Whitt, S.; Tobin, J. Science 1994, 264, 1927-1930.

(34) Ash, E. L.; Sudmeier, J. L.; De Fabo, E. C.; Bachovchin, W. W. Science 1997, 278, 1128-1132.

(35) Craik, C. S.; Roczniak, S.; Largman, C.; Rutter, W. J. Science 1987, 237, 909-913.

(36) Mao, L.; Luo, S.; Huang, Q.; Lu, J. Sci. Rep. 2013, 3, 3126.

(37) Gonzalez-Perez, D.; Garcia-Ruiz, E.; Ruiz-Duenas, F. J.; Martinez, A. T.; Alcalde, M. ACS Catal. 2014, 4, 3891-3901.

(38) Morikubo, N.; Fukuda, Y.; Ohtake, K.; Shinya, N.; Kiga, D.; Sakamoto, K.; Asanuma, M.; Hirota, H.; Yokoyama, S.; Hoshino, T. J. Am. Chem. Soc. 2006, 128, 13184-13194.

(39) Minnihan, E. C.; Young, D. D.; Shultz, P. G.; Stubbe, J. J. Am. Chem. Soc. 2011, 133, 15942-15945.

(40) Aldag, C.; Gromov, I. A.; Garcia-Rubio, I.; von Koenig, K.; Schlichting, I.; Jaun, B.; Hilvert, D. Proc. Natl. Acad. Sci. U. S. A. 2009, 106, 5481-5486.

(41) Vandemeulebroucke, A.; Aldag, C.; Stiebritz, M. T.; Reiher, M.; Hilvert, D. Biochemistry 2015, 54, 6692-6703.

(42) Jiang, Y.; Sivaramakrishnan, S.; Hayashi, T.; Cohen, S.; MoenneLoccoz, P.; Shaik, S.; Ortiz de Montellano, P. R. Angew. Chem., Int. Ed. 2009, 48, 7193-7195.

(43) Hilvert, D. Annu. Rev. Biochem. 2013, 82, 447-470.

(44) Karplus, P. A.; Diederichs, K. Science 2012, 336, 1030-1033.

(45) Murshudov, G. N.; Vagin, A. A.; Lebedev, A.; Wilson, K. S.; Dodson, E. J. Acta Crystallogr., Sect. D: Biol. Crystallogr. 1999, 55, 247255.

(46) Emsley, P.; Cowtan, K. Acta Crystallogr., Sect. D: Biol. Crystallogr. 2004, 60, 2126-2132. 